\title{
Confinement effects on optical phonons in spherical, rod-, and tetrapod-shaped nanocrystals detected by Raman spectroscopy
}

\author{
Concetta Nobile $^{1}$, Stefan Kudera ${ }^{1}$, Angela Fiore ${ }^{1}$, Luigi Carbone ${ }^{1}$, Gerwin Chilla ${ }^{2}$, \\ Tobias Kipp ${ }^{2}$, Detlef Heitmann ${ }^{2}$, Roberto Cingolani ${ }^{1}$, Liberato Manna ${ }^{1}$, \\ and Roman Krahne ${ }^{*}, 1$ \\ 1 National Nanotechnology Laboratory of CNR-INFM, Via per Arnesano km5, 73100 Lecce, Italy
2 Institute of Applied Physics, University of Hamburg, 20355 Hamburg, Germany
}

Received 30 July 2006, revised 7 November 2006, accepted 13 November 2006 Published online 6 February 2007

PACS 63.22.+m, 71.35.Ji, 73.22.-f, 78.30.Fs, 78,67.Bf

Spherical, rod- and tetrapod shaped CdSe nanocrystals are investigated by Raman spectroscopy and the longitudinal-optical and surface optical phonons are observed. We find that the position of the longitudinal-optical phonon slightly red-shifts with decreasing diameter, whereas the position of the surface optical phonon depends significantly on diameter and length of the rods or the tetrapod arms.

(ㄷ) 2007 WILEY-VCH Verlag GmbH \& Co. KGaA, Weinheim

Chemical synthesis has achieved remarkable control over the size and shape of colloidal nanocrystals leading to spheres [1], rods [2-4], and branched nanostructures [5-7]. The shape of colloidal nanocrystals has strong effects on their optical and electronic properties, for example, in spherical nanocrystals the electronic bandgap, the resonant Stokes shift, and the optical phonon excitation energies depend on the nanocrystal diameter. Rod-shaped nanocrystals provide a uniaxial symmetry that leads to laterally and longitudinally confined vibronic excitations [8-10]. Tetrapods represent an even more complex geometry where four arms branch out from a central core that results in novel electronic and optical properties [11-13]. Optical phonons in nanocrystal spheres and rods have been studied experimentally and theoretically [14-18]. In spherical nanocrystals the confinement leads to a red-shift and broadening of the longitudinal-optical (LO) phonon mode [19], and to the observation of surface-optical (SO) phonons $[20,21]$. Phonon excitations in nanorods and tetrapods can be treated in a nanowire picture $[8,9$, $13,22-25]$ where the confinement depends on the length and diameter of the nanocrystal structure.

Colloidal CdSe nanospheres, nanorods, and tetrapods in wurtzite crystalline structure were fabricated by chemical synthesis in a hot mixture of surfactants following a published approach $[4,5,7,26]$. The unique axis of the wurtzite crystalline structure enables anisotropic growth due to the different chemical reactivity and growth rate of the lateral and longitudinal crystal facets. The transmission electron microscopy images of the three samples with different shapes that will be investigated in the following are shown in Fig. 1. The Raman experiments were performed using a diode laser at $532 \mathrm{~nm}$ wavelength that was coupled into an optical microscope setup and detected by a triple Raman spectrometer (Dilor XY) and a charge coupled device camera. The nanocrystals were casted from solution onto a Si substrate, dried under nitrogen flow and mounted into an optical cryostat.

\footnotetext{
${ }^{*}$ Corresponding author: e-mail: roman.krahne@unile.it
} 



Fig. 1 Transmission electron microscopy images of CdSe (a) dots with $8 \mathrm{~nm}$ diameter, (b) rods with $5 / 25 \mathrm{~nm}$ diameter/length and (c) tetrapods with $6 / 40 \mathrm{~nm}$ arm diameter and length.

Figure 2 shows the absorption and luminescence spectra of the three samples that are displayed in Fig. 1, recorded at room temperature and in solution. The well defined level structure in the dots leads to a series of distinct peaks in the absorption spectrum. In rods and tetrapods the more complicated electronic structure results in overlapping peaks in their absortion spectra. The fluorescence peaks show a blue-shift with decreasing diameter of the nanocrystals, confirming that the diameter is the dominant parameter for the optical excitations. The fluorescence peaks are red shifted from the lowest absorption peaks by the Stokes shift, which increases with decreasing nanocrystal diameter and rising nanocrystal shape complexity. The particular geometry of the tetrapods leads to a double peak structure in their fluorescence $[12,27]$. Here we observe a large peak at $2 \mathrm{eV}$ that results from recombination of the exciton ground state and a small peak at the high energy side at $2.2 \mathrm{eV}$ peak that we believe originates from recombination of the first excited exciton state.

Figure 3 shows Raman spectra of the dots, tetrapods and rods recorded at $T=13 \mathrm{~K}$ with a power density of $1 \mathrm{~mW} / \mu \mathrm{m}^{2}$. We observe an asymmetric excitation and its higher scattering orders (not shown) for

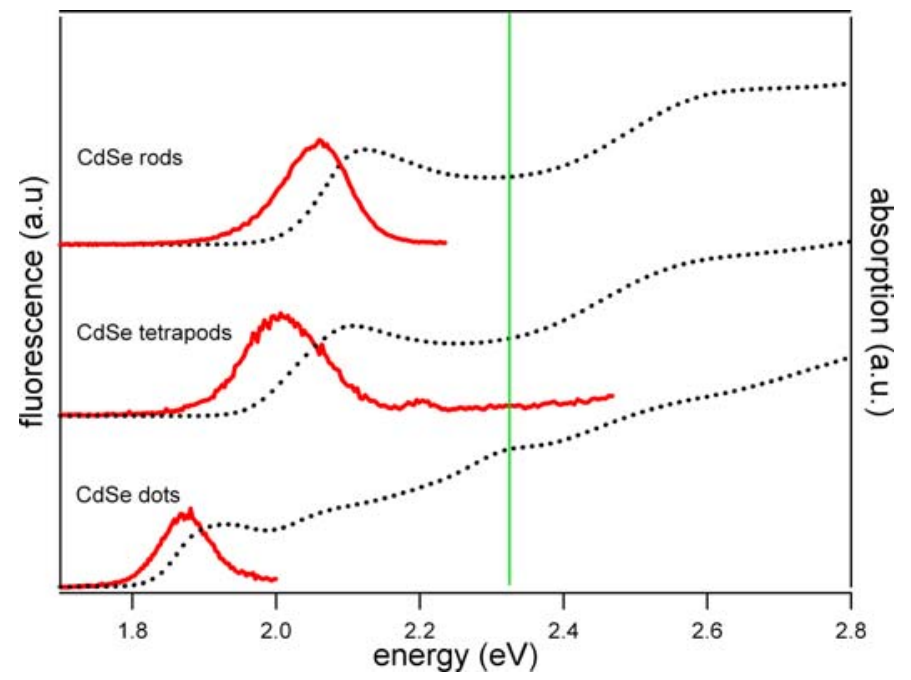

Fig. 2 (online colour at: www.pss-a.com) Fluorescence (solid lines) and absorption spectra (dashed lines) of the dot, rod, and tetrapod samples under investigation. The fluorescence of the tetrapods shows a second peak (at $2.2 \mathrm{eV}$ ), whereas in the case of dots and rods only a single peak is observed. The green vertical line marks the laser excitation energy for the Raman experiments. 


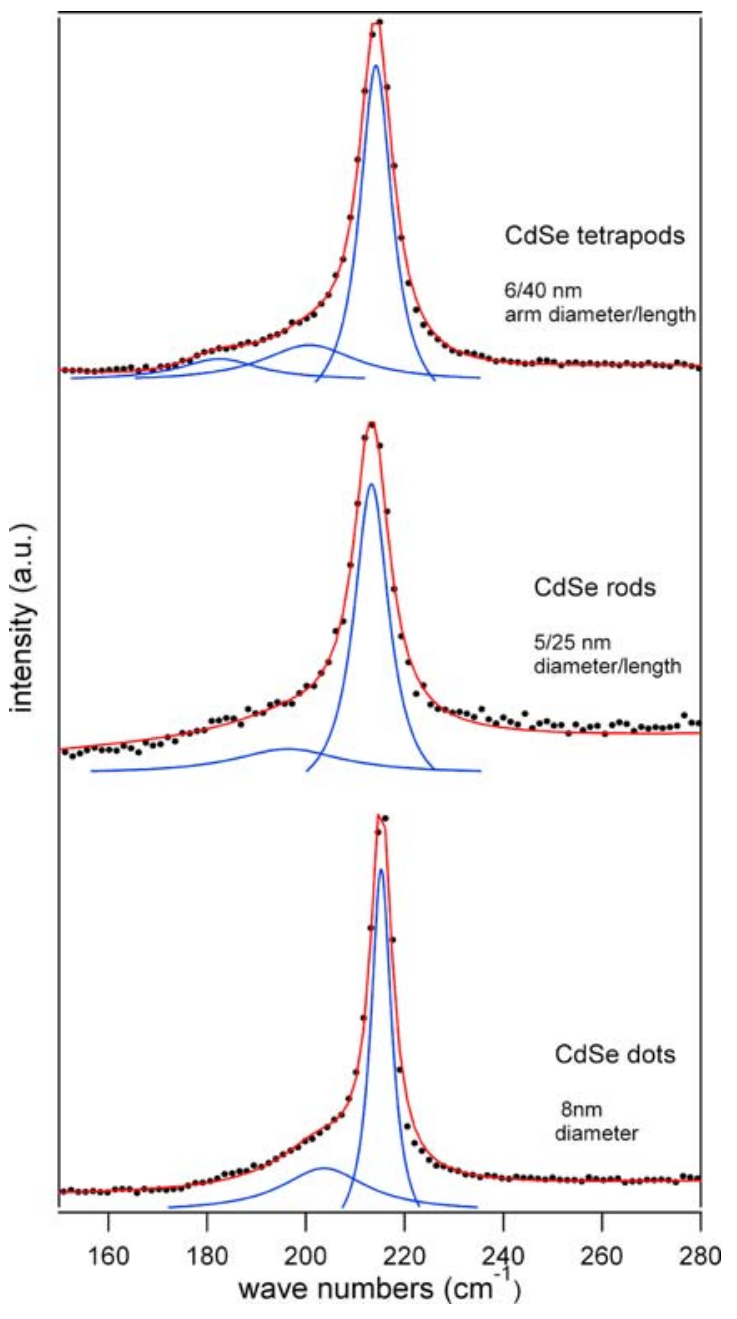

Fig. 3 (online colour at: www.pss-a.com) Raman spectra of nanocrystals with different shape. We observe the LO phonon modes at $215 \mathrm{~cm}^{-1}$ for dots, $213 \mathrm{~cm}^{-1}$ for rods and $214 \mathrm{~cm}^{-1}$ for tetrapods, showing a trend that a decreasing nanocrystal diameter leads to an increasing red shift of the LO phonon frequency. The SO phonon modes at the low energy side of the LO phonon peak are sensitive to the aspect ratio of the nanocrystal shape. The thin solid lines show best Lorentzian fits to the data.

all nanocrystal shapes. The high energy peaks of this excitation can be attributed to the LO phonon scattering. The Lorentzian fits to the data reveal well defined peaks at $215 \mathrm{~cm}^{-1}$ for dots, $214 \mathrm{~cm}^{-1}$ for tetrapods and $213 \mathrm{~cm}^{-1}$ for rods showing a trend that the LO phonon shifts to lower energy with decreasing nanocrystal diameter, as it was observed in experiments on spheres with different diameters. The low energy side of the excitation can be fitted with peaks at $203 \mathrm{~cm}^{-1}$ for dots, $196 \mathrm{~cm}^{-1}$ for rods, and for tetrapods with a double peak located at 185 and $200 \mathrm{~cm}^{-1}$. The low energy peak values can be modeled by the SO phonon energies for the respective geometries. To calculate the SO phonon energies of spherical nanocrystals we apply the dielectric continuum model of Ruppin and Englman [28]

$$
\omega_{\mathrm{SO}}^{2}=\omega_{\mathrm{TO}}^{2} \frac{\varepsilon_{0}+\varepsilon_{\mathrm{M}}(l+1) / l}{\varepsilon_{\infty}+\varepsilon_{\mathrm{M}}(l+1) / l} ; \quad l=1,2,3 \ldots,
$$

where $\varepsilon_{\mathrm{M}}=1$ (surrounding medium dielectric constant) for vacuum, $\varepsilon_{\infty}$ and $\varepsilon_{0}$ are the bulk CdSe high frequency and static dielectric constants, respectively, and $\omega_{\mathrm{TO}}=173 \mathrm{~cm}^{-1}$ is the frequency of the transverse-optical phonon. This approach yields for the SO phonon ground mode $(l=1)$ of $\mathrm{CdSe}$ spheres $\omega_{\mathrm{SO}}=205 \mathrm{~cm}^{-1}$, in good agreement with the low energy peak of the dot sample located at $203 \mathrm{~cm}^{-1}$. 
For the rods and tetrapods we follow the approach of Gupta and coworkers [9] for surface optical (SO) phonons in nanowires [13]:

$$
\omega_{\mathrm{SO}}^{2}=\omega_{\mathrm{TO}}^{2}+\frac{\varpi_{p}^{2}}{\varepsilon_{\infty}+\varepsilon_{\mathrm{M}} f(x)} ; \quad x=q \cdot d / 2,
$$

where $\varpi_{p}^{2}=\varepsilon_{\infty}\left(\omega_{\mathrm{LO}}^{2}-\omega_{\mathrm{TO}}^{2}\right)$ is the screened ion-plasma frequency, $f(x)=\left(I_{0}(x) K_{1}(x)\right) /\left(I_{1}(x) K_{0}(x)\right)$ (with $I$ and $K$ Bessel functions), and $d$ is the rod (or tetrapod arm) diameter. We can take the length of the rods (or the tetrapod arms) as the longitudinal symmetry breaking mechanism that leads to $q=2 \pi / l$. For the tetrapod samples the SO phonon is calculated at $\omega_{\mathrm{SO}}=185 \mathrm{~cm}^{-1}(5 / 40 \mathrm{~nm}$ diameter/length), and for the rods $\omega_{\mathrm{SO}}=195 \mathrm{~cm}^{-1}(5 / 22 \mathrm{~nm}$ diameter/length), also in very good agreement with our data. The signal that we observe in the tetrapods in the regime between the SO and the LO phonon could be due to higher harmonics of the surface modes, or could originate from LO or TO phonon mode splittings due to the wire geometry [13].

In conclusion, we measured the LO and SO phonons in CdSe nanocrystals with different shapes. We find that the LO phonon frequency depends dominantly on the nanocrystal diamenter, whereas the SO phonon is also sensitive on the length of the nanocrystal rods.

Acknowledgements The authors gratefully acknowledge the support by the SA-NANO European project (Contract No. STRP013698), by the MIUR-FIRB and MIUR 297 (Contract No. 13587) projects.

\section{References}

[1] A. P. Alivisatos, Science 271, 933 (1996).

[2] S. Kan, T. Mokari, E. Rothenberg et al., Nature Mater. 2, 155 (2003).

[3] J. T. Hu, L. S. Li, W. D. Yang et al., Science 292, 2060 (2001).

[4] L. Manna, E. C. Scher, L. S. Li et al., J. Am. Chem. Soc. 124, 7136 (2002).

[5] L. Manna, D. J. Milliron, A. Meisel et al., Nature Mater. 2, 382 (2003).

[6] K. A. Dick, K. Deppert, M. W. Larsson et al., Nature Mater. 3, 380 (2004).

[7] L. Carbone, S. Kudera, E. Carlino et al., J. Am. Chem. Soc. 128, 748 (2006).

[8] G. D. Mahan, R. Gupta, Q. Xiong et al., Phys. Rev. B 68, 73402 (2003).

[9] R. Gupta, Q. Xiong, G. D. Mahan et al., Nano Lett. 3, 1745 (2003).

[10] Q. H. Xiong, J. G. Wang, O. Reese et al., Nano Lett. 4, 1991 (2004).

[11] Y. Cui, U. Banin, M. T. Bjork et al., Nano Lett. 5, 1519 (2005).

[12] D. Tarì, M. De Giorgi, F. Della Sala et al., Appl. Phys. Lett. 87, 224101 (2005).

[13] R. Krahne, G. Chilla, C. Schüller et al., Nano Lett. 6, 478 (2006).

[14] E. Roca, C. Tralleroginer, and M. Cardona, Phys. Rev. B 49, 13704 (1994).

[15] M. P. Chamberlain, C. Tralleroginer, and M. Cardona, Phys. Rev. B 51, 1680 (1995).

[16] V. A. Fonoberov and A. A. Balandin, Phys. Rev. B 70, 233205 (2004).

[17] V. A. Fonoberov and A. A. Balandin, J. Phys.: Condens. Matter 17, 1085 (2005).

[18] V. A. Fonoberov and A. A. Balandin, J. Nanoelectron. Optoelectron. 1, 19 (2006).

[19] K. A. Alim, V. A. Fonoberov, and A. A. Balandin, Appl. Phys. Lett. 86, 053103 (2005).

[20] A. Roy and A. K. Sood, Phys. Rev. B 53, 12127 (1996).

[21] A. M. dePaula, L. C. Barbosa, C. H. B. Cruz et al., Appl. Phys. Lett. 69, 357 (1996).

[22] Q. H. Xiong, G. Chen, J. D. Acord et al., Nano Lett. 4, 1663 (2004).

[23] Q. H. Xiong, R. Gupta, K. W. Adu et al., J. Nanosci. Nanotechnol. 3, 335 (2003).

[24] T. Thonhauser and G. D. Mahan, Phys. Rev. B 69, 075213 (2004).

[25] T. Thonhauser and G. D. Mahan, Phys. Rev. B 71, 081307 (2005).

[26] Z. A. Peng and X. G. Peng, J. Am. Chem. Soc. 123, 183 (2001).

[27] R. Krahne, G. Chilla, C. Schüller et al., J. Nanoelectron. Optoelectron. 1, 104 (2006).

[28] R. Ruppin and R. Englman, Rep. Prog. Phys. 33, 149 (1970). 